\section{European Medical Research Group (Meeting held on 4 June 1991)}

The European Medical Research Group met at the Medical Society of London, Lettsom House, on 4 June 1991. The guest speaker was Dr K.D. Macrae, Reader in Statistics, Charing Cross \& Westminster Medical School, London, who gave a lecture entitled 'How to ensure your paper is rejected by the statistical referee'.

Following a period of questions and discussion, a poster session was held demonstrating the research in progress of some members of the Group. Their abstracts are published below.

\section{Changes in plasma and cerebrospinal fluid (CSF) beta-endorphin ( $\beta E P$ ) levels during surgery in humans. Comparison between general versus spinal anaesthesia (GA vs SA)}

\author{
A.I. Lykiardopoulou, N.J. Sarlis, P.K. Kaniaris, \\ M. Mykoniakis and R.A. Knight
}

\section{Charing Cross and Westminster Medical School, Charing Cross Hospital, Department of Medicine, Neuroendocrinology Unit, 5th Floor, Fulham Palace Road, London W6 8RF, UK, and University of Athens Medical School, Department of Experimental Pharmacology, 75 Mikras Asias Street, Goudi, Athens, GR11527, Greece.}

Surgical and anaesthetic differential activation of the neuroendocrine stress axis caused by regional spinal anaesthesia (SA) versus general anaesthesia (GA) has long been debated. The present study compared the plasma and CSF $\beta E P$ and plasma cortisol (F) levels before, during and following GA and SA.

With informed consent and Patients' Trial Ethics Committee approval, 14 male patients undergoing suprapubic prostatectomy were studied. Patients who suffered from neoplastic or systemic diseases or were under treatment with drugs interacting with the hypothalamopituitary unit were excluded. Seven of the patients were allocated to the GA group and the rest to the SA group. Average age was: GA group $=70 \pm 5$ years and SA group $=73 \pm 5$ years (N.S.). The duration of the operation was $85 \pm 5 \mathrm{~min}$ in the GA group vs $84 \pm 4 \mathrm{~min}$ in the SA group (N.S.). All patients were selected to be of ASA II and III risk status. They were all given diazepam $10 \mathrm{mg}$ p.o. preoperatively. The GA group patients received thiopental $5 \mathrm{mg} / \mathrm{kg}$, succinylcholine $1 \mathrm{mg} / \mathrm{kg}$, fentanyl $2 \mu \mathrm{g} / \mathrm{kg}$, pancuronium $0.1 \mathrm{mg} / \mathrm{kg}$ and inhaled an $\mathrm{O}_{2} / \mathrm{NO}_{2}$ $2: 4$ mixture and fluothane $0.5 \%$. Their tracheas were intubated following spraying with lidocaine. The SA group patients had a spinal tap at the level L3-L4 and $2 \mathrm{ml}$ lidocaine $5 \%$ and $1 \mathrm{ml}$ bupivacaine $0.5 \%$ were infused. The level of analgesia was neurologically assessed to be T8-T10. These patients received no inhalation or i.v. supplementation. Both groups were infused i.v. with Ringer's lactate and no vasospastic amines or blood transfusions were given. Plasma $\beta E P$ and $F$ samples were drawn through an indwelling venous catheter immediately prior to the entry to the operating theatre, $45 \mathrm{~min}$ after induction and at the end of the operation ( $\mathrm{ca} 90 \mathrm{~min}$ post-induction). CSF samples were taken by spinal tap before and just after the operation. Plasma and CSF $\beta E P$ levels were determined by Immunonuclear Standard (Minnesota, USA) BEP RIA kits. F levels were measured by fluorescence polarization immunobiological method using a TDX-Abbott analyser.

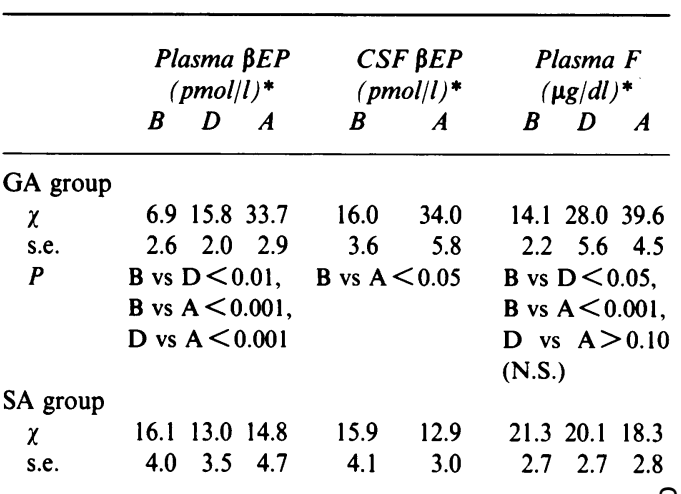

All changes from control values $(B=$ before) were N.SO $(P<0.10) .{ }^{*} \mathrm{~B}=$ before; $\mathrm{D}=$ during; $\mathrm{A}=$ after.

Association of plasma $\beta E P$ with stressful situations has been thoroughly documented. Our results implicate $G \vec{A}$ as being more stressful than SA, as measured by simultaneous sampling of both plasma and CSF $\beta$ EP and plasma cortisol in humans.

\section{Assessment of the Riyadh Intensive Care Programme as applied to a mortality analysis of a UK teaching hospital intensive care unit}

\section{S. Jacobs, A. Arnold, P.A. Clyburn and B.A. Willis}

Department of Intensive Care/Anaesthesia,
University Hospital of Wales, Cardiff, UK.

A computerized system of prediction of death using the Riyadh Intensive Care Programme (RIP) was applied retrospectively over a 17 month period to 1,155 patients admitted to the Intensive Care Unit (ICU). RIP is based on trend analysis of daily organ failure scores and the following variables were recorded daily to calculate the scores. Deviation from normal of 11 acute physiological variables (rectal temperatures, mean arterial pressure, heart rate, oxygenation, arterial $\mathrm{pH}$, serum sodium, potassium, creatinine, haematocrit and white cell count), the Glasgow Coma score, the patient's age and the presence of defined chronic disease. The system predicted 
death in only $15 \%$ of our total ICU mortality during this period (31/209) and 3 patients were falsely predicted to die. The predictive power of the programme demonstrated a sensitivity of $14.8 \%$ and a specifity of $99.8 \%$. The significant event of false predictions of death may have been related to our change in antibiotic policy during this period. We cannot recommend the use of computerized systems of prediction of death for ICU patients to withdraw therapy without careful explanation of its significance and dangers.

\section{A retrospective study of myocardial infarction occurring in elderly patients managed in a coronary care unit}

\author{
E.C. Mulkerrin, G.C. Morris, R. Dewar, \\ C. Condon, J. Curran, A.J. Bayer and M.S. Laher
}

\section{Department of General Medicine, James Connolly Memorial Hospital, Dublin, Ireland, and University Department of Geriatric Medicine, Cardiff, UK.}

Myocardial infarction is a major cause of morbidity and mortality, particularly in the elderly. Before the advent of Coronary Care Unit monitoring, the mortality of acute myocardial infarction was reported to increase dramatically with increasing age. ${ }^{1}$ We have undertaken a retrospective analysis of the presentation, clinical course, complications and outcome of 204 consecutive patients admitted to a Coronary Care Unit with proven acute myocardial infarction between 1984 and 1988.

Of the 260 consecutive cases, adequate data was obtained in 204 cases and we report the results of its analysis. The patients were divided into those over 75 years $(n=84)$ and those between 65 and 75 years $(n=120)$ at admission. Follow-up data obtained from hospital outpatient notes when possible and those lost to follow-up were sent a postal questionnaire regarding subsequent hospital admissions and current symptomatology.

When compared to those patients aged 65-75 years, the older group contained fewer current smokers $(P<$ 0.02 ). They were less likely to present with chest pain $(P<0.05)$. Although the evidence of cardiac arrhythmias and lignocaine therapy was similar, other agents were more frequently used in older patients $(P<0.01)$ and these were effective in $88 \%$ of cases. Congestive cardiac failure occurred with similar frequency in both groups but the older group more often required inotropic agents.
Cardiac enzyme release was significantly lower in the older group $(P<0.001)$.

Although the groups differed significantly in terms of clinical presentation they had similar complication rates, morbidity and mortality.

\section{References}

1. Biorck, G.J. Course and prognosis in some cardiac diseases Chron Dis 1962, 15: 9.

\section{Echocardiography in the assessment of cardiac failure in the elderly}

\section{R.I. Dewar and E. Mulkerrin \\ University Department of Geriatrics, Cardiff Royal Infirmary, Cardiff CF2 1SZ, UK.}

Cardiac failure with preserved systolic function is well described and may have implications for management. As diastolic function changes with increasing age, recognition of these patients may be important in the elderly. This study was designed to use echocardiography to determine the systolic and diastolic function in a series of elderly patients with heart failure.

Twenty-six consecutive inpatients admitted to an acute geriatric ward, who fulfilled the Boston Criteria for definite heart failure, underwent an echocardiographic study. Measures included left ventricular ejection fraction ('normal' LVEF $\geqslant 50 \%$ ), left ventricular wall thickness (LVWT) and LV diastolic function, early filling deceleration rate from the mitral inflow Doppler (ERDR). The echocardiographic data were compared between subgroups using the unpaired $t$-test.

The 26 patients ( 15 female) had a mean age of 79 (67-92 years). Twelve had normal systolic function. Comparison of the subgroups with normal or abnormal systolic function revealed a significant difference in diastolic function, EFDR $367( \pm 257)$ and $628( \pm 229)$ ms-2 respectively $(P=0.01)$. The ages and wall thickness measurements of the subgroups were not different.

We conclude that, in a series of elderly patients with heart failure, $46 \%$ had normal systolic function, and this group showed evidence of significant diastolic dysfunction. 